\title{
Condylar Translation and the Function of the Superficial Masseter Muscle in the Rhesus Monkey (M. mulatta) ${ }^{1}$
}

\author{
DAVID S. CARLSON \\ Center for Human Growth and Development, The University of Michigan, \\ Ann Arbor, Michigan 48109
}

\author{
KEY WORDS Muscle . Mastication . TMJ + Biomechanics · \\ Rhesus monkey
}

\begin{abstract}
The relationship between translation of the mandibular condyle during symmetrical mandibular rotation, i.e., symmetrical jaw depression and elevation, and the function of the superficial masseter muscle was examined in light of relative torque and the length-tension relationship for muscle. Lateral cephalograms of live adult rhesus monkeys (Macaca mulatta) were analyzed using two models: (1) Model A, normal symmetrical jaw rotation accompanied by condylar translation; and (2) Model B, mandibular rotation about an axis fixed at the position of the condyles during centric occlusion.

The decrease in relative torque and the excursion of the superficial masseter at mouth-open positions are significantly greater in Model B than in Model A. Symmetrical rotation of the jaw about a fixed axis would result in a $35 \%$ greater loss of maximum producible tension at maximum gape than rotation associated with condylar translation. These results suggest that condylar translation during mandibular depression and elevation functions to minimize reduction in relative torque and excursion of superficial masseter muscle, thereby maintaining optimal potential for exerting maximum tension during jaw closure.
\end{abstract}

The mechanics of jaw movement in man and other primates is a topic of widespread concern among physical anthropologists and paleontologists (Gingerich, '71; Barbenel, '72; Grant, '73; Hiiemae and Kay, '73; Kay and Hiiemae, '74; Hylander, '75a; Isaacson et al., '75). The rationale behind this concern is simple, and is based upon a close relationship be tween anatomical form and function. Numerous studies emphasize this relationship in terms of the biomechanics of mastication, both as a means of explaining morphological variation among extant forms (Moffett, '66; Gilbe, '73; Du Brul, '74; Herring and Herring, 74) and as a means of accounting for evolutionary change in the vertebrate skull (Smith and Savage, '59; Crompton, '63; Scapino, '72; Du Brul, '74; Wolpoff, '75).

Much of the recent attention on jaw mechanics focuses on two related questions: (1) can the mandible be most accurately characterized as a lever or as a "link"? and (2) what is the adaptive and mechanical significance of each of the several general types of masticatory complexes? The first point is summarized and reviewed most effectively in a recent article by Hylander ('75a), who concludes that the non-lever ("link") hypothesis of jaw function in man is "ill-founded," and that the human mandible can be most accurately viewed as a lever for purposes of analyzing masticatory function. This conclusion is in basic agreement with most previous investigations of mammalian jaw mechanics (Davis, '55; Smith and Savage, '59; Turnbull, '70; Hiiemae, '71; Hoshi, '71). The functional significance of the general types of jaw mechanics (e.g., between herbivores and carnivores), is resolved in large part. Turnbull ('70) and Noble ('73), for example, provide excellent summaries of the comparative anatomical and paleontological evidence bearing on this problem, and have succeeded in identifying two or three major

\footnotetext{
${ }^{1}$ This research was supported in part by United States Public Health Service Grants DE-04227 and DE.03610, and by NIH Post. Doctoral Fellowships DE-013696 and DE-05101.
} 
types of jaw function in mammals, each closely related to its specific ecological and behavioral attributes. Numerous recent studies of jaw function help to clarify further the pattern of jaw mechanics found in man and other primates (Gibbs et al., '71; McNamara, '74; Brill and Tryde, '74; Griffin and Malor, '74; Graf, '75).

Despite the above efforts, a number of methodological and empirical questions remain concerning the function of the masticatory apparatus and its specific adaptive significance. As noted by Hylander ('75a), for example, the method of approach to a static biomechanical analysis of the mandible as well as the significance of the method in obtaining accurate empirical data on mastication is open to question. Specifically, most analyses of jaw mechanics consider the mandibular axis of rotation to be located at the condyles. This is despite the fact that in many mammalian forms, including primates, the mandibular condyle is translated anteriorly during jaw opening, thus precluding the possibility that the true axis of rotation resides at the condyle during all phases of jaw movement. At first glance this appears to be a major inconsistency, but as seen below, consideration of the axis of rotation at the condyles is simply a methodological convention which many believe does not violate any known factors affecting masticatory function. It is this assumption, however, which is called into question.

At least two theories have been advanced to account for the adaptive significance of a variable axis of mandibular rotation. Moss ('59; also see Ricketts,'75) suggested that the mandible rotates about the mandibular foramen, and that this represents an adaptation to reduce the amount of movement about this region in order to minimize the potential for trauma of the inferior alveolar nerve. While it is empirically demonstrable that the axis of mandibular rotation closely approximates the position of the mandibular foramen, however, it is impossible to determine whether this relationship is a cause or an effect of the axis location.

Grant ('73) attributed the adaptive significance of a variable axis of rotation ("instantaneous center of rotation" - ICR) to the biomechanical efficiency of the muscles of mastication. He argued that the forces generated by the muscles of mastication vary greatly depending on the position of the mandibular axis of rotation, such that a fixed axis at the condyles is much less efficient than a variable axis of rotation. However, Stern ('74) correctly notes that the relative contribution of each of the muscles of mastication during jaw function is not affected by the location of the axis of rotation. As long as the axis is outside the TMJ the reaction force at the condyles must be taken into consideration in any analysis of forces acting on the mandible to affect jaw opening or closure. It is simply for ease of computation that the condyle is considered the axis of rotation since this approach eliminates the need to account for joint reaction in the analysis of rotatory equilibrium (Stern, '74: p. 109).

The above arguments led Hylander to conclude that "the supposed instantaneous center of rotation is apparently of little use in the determination of muscle function" ('75: p. 237). It should be emphasized, however, that Stern's comments on the significance of a variable axis of rotation are relevant only to a consideration of muscle torque, and that torque is only one component affecting the efficiency of muscle function. Recent studies by Rayne and Crawford ('72) and by Herring and Herring ('74) stress that parameters of muscle function other than just torque must be considered in order to establish a more complete understanding of jaw function. While relative torque during mandibular function is unaffected by the location of the axis of mandibular rotation, the same statement cannot yet be made concerning the effect of a variable axis of rotation on overall muscle function.

\section{PURPOSE}

The purpose of this analysis is to investigate the relationship between the function of the superficial masseter muscle and the mechanics of the TMJ in the rhesus monkey. The following hypothesis is presented: the variable axis of mandibular rotation in primates represents an adaptation for providing maximum gape with minimal excursion and thus minimal loss of efficiency of the superficial masseter muscle.

Analysis of jaw function focuses on symmetrical mandibular rotation, i.e., depression and elevation in the sagittal plane without lateral translation. This approach is limited to some extent since it focuses on only one 
elevator of the mandible and because the primate jaw does not normally function symmetrically during the masticatory cycle (Graber, '72; Hiiemae and Kay, '73; Hylander, '75a). However, the primate mandible probably does function relatively symmetrically during depression associated with gape, as occurs during a threat display or preparation for forceful biting during aggressive behavior, as well as during elevation associated with incisal or bilateral biting. Both these features may be critical to an understanding of the selective factors which affected the evolution of the primate masticatory apparatus.

\section{MANDIBULAR MOVEMENT}

The functional movements of the mandible have been studied extensively in man (Gibbs et al., '71; Graber, '72; Griffin and Malor, '74), and knowledge in this area is relatively complete. Although recent studies of jaw function in some non-human primates indicate interspecies variation (Hiiemae and Kay, '73; Kay and Hiiemae, '74), electromyographic analysis of orofacial muscles during maintenance of posture, deglutition, and mastication suggest close functional similarities between man and $M$. mulatta (McNamara, '72, '74; McNamara and Moyers, '73). These similarities are likely to be most apparent during symmetrical mandibular rotation.

Mandibular movement is defined in terms of several distinct static mandibular positions. Three are relevant to the present investigation.

1. Centric occlusion is a tooth-to-tooth relationship of the mandible to the maxilla in which there is maximal intercuspation of the opposing teeth (Ramfjord and Ash., '71). This position is the same as habitual occlusion and intercuspal position in normal occlusion, i.e., in the absence of malocclusion (Graber, '72).

2. The normal postural position of the mandible is referred to as its rest position. With the exception of the tonic activity in the anterior and posterior components of the temporalis muscle to resist the effects of gravity, neuromuscular activity is minimal in the elevators of the mandible at the rest position during normal posture (Latif, '57; Møller, '66, '74). According to Garnick and Ramfjord ('62), the mean interocclusal distance at the rest position in man is between $1.3 \mathrm{~mm}$ and $1.7 \mathrm{~mm}$.
3. Maximum gape refers to the position of the mandible when opened to the greatest degree possible without inducing trauma to the TMJ or other oral structures.

\section{Mandibular rotation}

As noted previously, the primate mandible does not normally rotate about a fixed axis at the mandibular condyles. Rather, the rotation of the mandible from the occlusal or rest positions through maximum gape during symmetrical jaw opening, or the reverse during symmetrical jaw closure, involves two distinct rotations made possible by the anatomy of the TMJ (Hjorsjö, '55). The mandibular condyles rotate both about themselves and about the articular eminence during jaw opening, resulting in a rotation of the mandible to an open position and a translation of the condyles anteriorly and inferiorly along the posterior slope of the articular eminence. At maximum gape the condyles sit directly on or slightly anterior to the peak of the articular eminence. Symmetrical jaw closure involves the exact opposite process. The condyles rotate posteriorly while at the same time being translated posteriorly and superiorly along the posterior slope of the articular eminence, finally occupying the glenoid fossae as centric occlusion is approached.

\section{MUSCLE MECHANICS}

It is beyond the scope of this paper to dis cuss the physiology of muscle contraction in detail (see Gans and Bock, '65; Carlson and Wilkie, ' 74 for discussions of pertinent muscle physiology). However, a major parameter of muscle physiology, the relationship between muscle length and producible tension, is critical to a consideration of the stated hypothesis on jaw function.

\section{Length-tension relationship}

Two of the most significant features characterizing muscle are its extensibility and its ability to exert tension (contract) from a wide range of excursion lengths. However, it is critical to note that the maximum level of tension capable of being exerted by a contracting muscle is dependent upon the length of the muscle at the time of stimulation. Thus, the capability of a muscle to generate force and carry out a given function can vary greatly depending on the distance between its skele- 


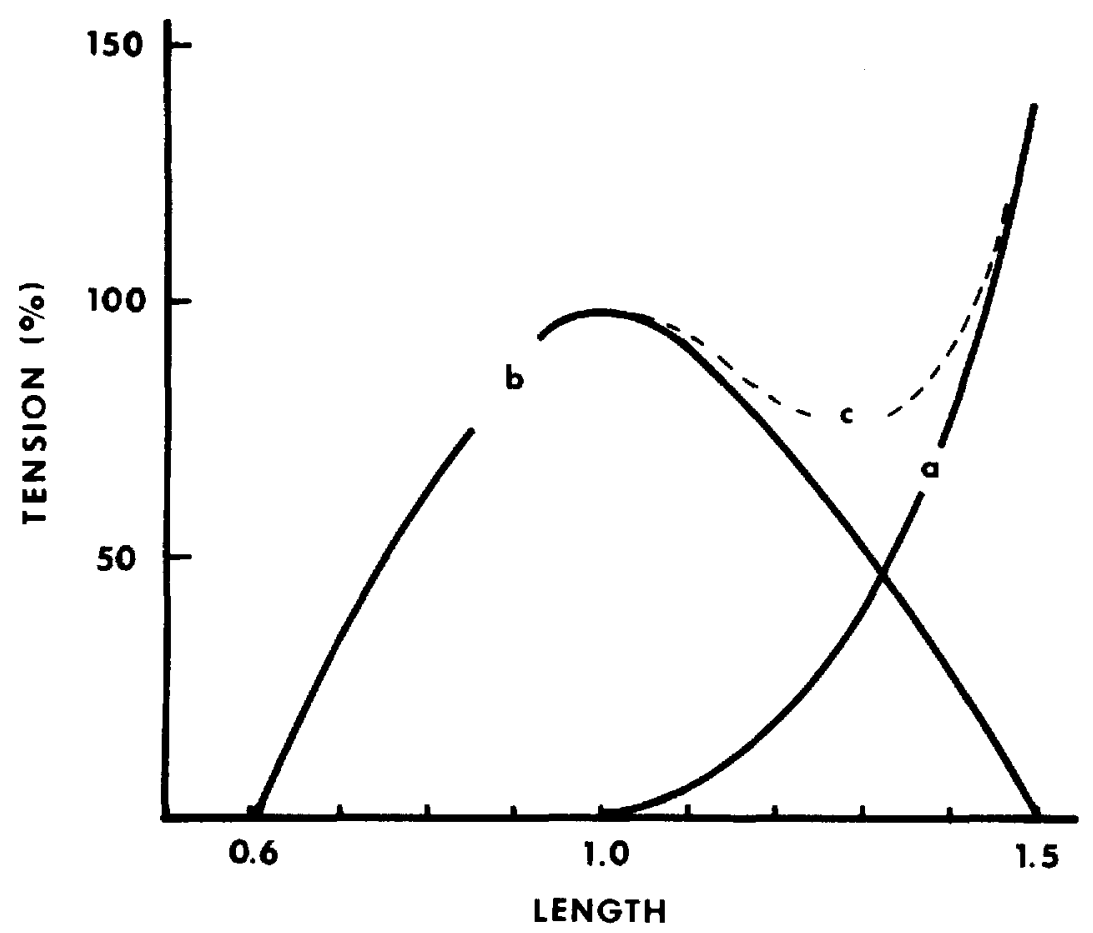

Fig. 1 Length-tension diagram for an entire muscle. Muscle length is given as a function of its resting length $\left(L_{1.0}\right)$. Tension is indicated along the ordinate as a percent of the maximum producible force upon complete tetanic stimulation. a, muscle at rest and stretched passively; $b$, tension developediduring maximum tetanic stimulation; $c$, total tension produced during maximal stimulation (sum of a and b). Note that producible tension (b) is greatest at $\mathrm{L}_{10}(100 \%)$, and that it drops off relatively abruptly at lengths less than or greater than resting length. (Adapted from Hill, '53: p. 113).

tal origin and insertion at the onset of stimulation by the nervous system.

Skeletal muscle fibers are capable of producing maximum tetanic tension at their resting length $\left(\mathrm{L}_{1.0}\right)$, where there is the greatest degree of overlap of contractile proteins within each myofibril. Overlap between the actin and myosin filaments decreases as muscles are stretched, resulting in a decrease in the level of tension capable of being produced by each fiber upon stimulation and an increase in the amount of time required to develop tension (Hill, '53). Potential tension also decreases when fibers are stimulated at lengths shorter than their resting length, probably due to a less efficient stimulation of the fiber (Carlson and Wilkie, '74). Although the presence of tendon and other connective tissue and fiber orientation tends to complicate the length-tension relationship for whole muscles, once these factors are taken into account the same general association between muscle length and maximal tetanic tension obtains (Hill, '53; Ramsey, '55; Gans and Bock, '65; Carlson and Wilkie, '74).

The length-tension relationship is summarized most effectively as a diagram relating percent producible tension and muscle length (fig. 1). According to Ramsey ('55), a $20 \%$ stretch in the length of muscle fibers $\left(L_{1.2}\right)$ results in approximately a $15 \%$ decrease in producible tension. The same increase in the length of an entire muscle results in approximately a $30 \%$ decrease in maximum tension (Hill, '53). Increased pennation and shorter fiber length of a muscle, characteristic of the muscles of mastication, tend to intensify the effects of muscle stretch, such that the percent decrease in producible tension is even more pronounced for each increment of increase in muscle length.

\section{MATERIALS AND METHODS}

Eleven adult female rhesus monkeys ( $\mathrm{Maca}$ - 


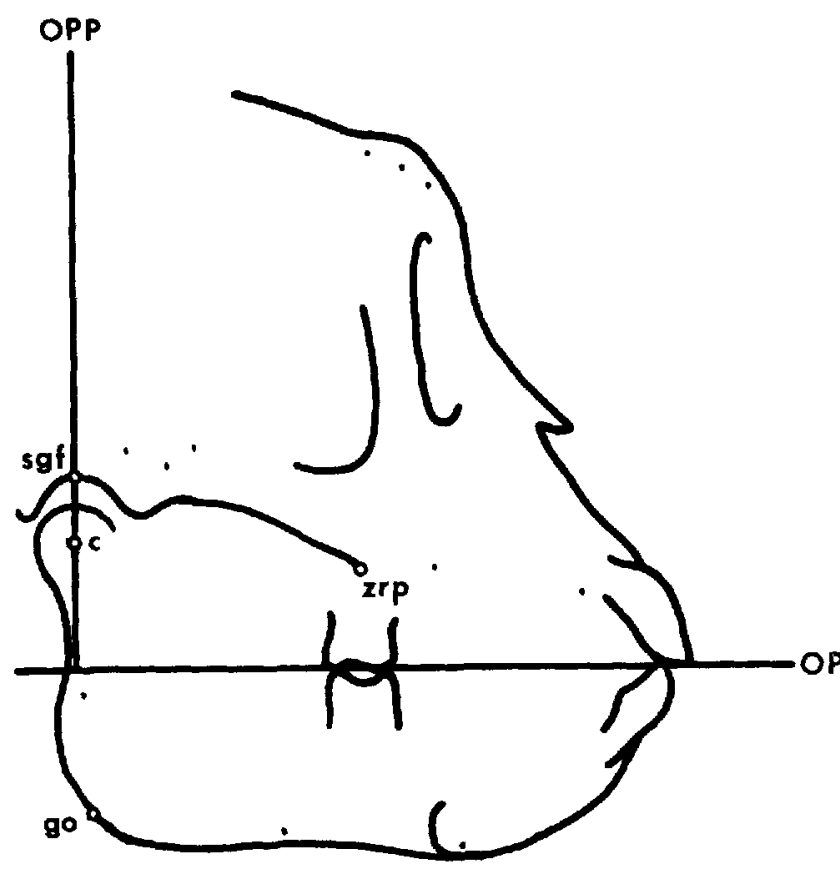

A

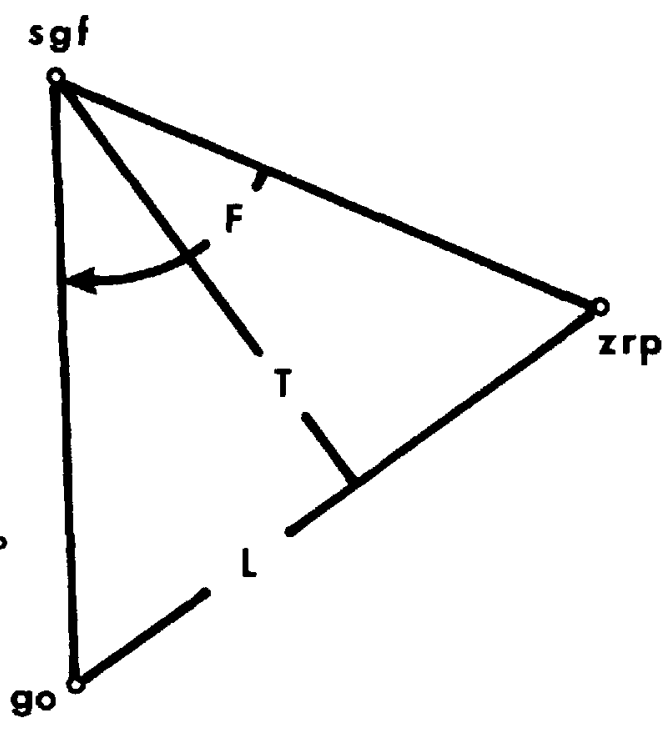

B

Fig. 2 Tracings of a lateral cephalogram of an adult rhesus monkey indicating: A, the four cephalometric points located on each radiograph, the maxillary occlusal plane (OP), and the maxillary occlusal plane perpendicular (OPP); and $B$, the three variables constructed from the cephalometric points. (See text for definitions.) Measurements were taken from cephalograms of each mandibular position for both Model A and Model B (fig. 3).

ca mulatta) were randomly selected for analysis. Each of the monkeys had been implanted with tantalum bone markers (McNamara, '72) and none had undergone any experimental intervention prior to this study.

Animals were sedated and their heads radiographed in a lateral projection. Cephalograms were taken of three mandibular positions. Position 1, centric occlusion, was obtained by maintaining the maxillary and mandibular dentition in maximal intercuspation using an elastic strap. Because of the close approximation of centric occlusion and the rest position (Garnick and Ramfjord, '62), Position 1 was considered to be the resting length of the superficial masseter muscle for purposes of analysis. Position 2 , a $3 \mathrm{~cm}$ incisal open bite, was obtained by inserting a plexiglass rod with a diameter of $3 \mathrm{~cm}$ between the upper and lower incisors. Position 3, maximum gape, was obtained by forcing the mouth open with a Weitlaner retractor.
The method for evaluating jaw movement and muscle excursion was adapted in part from a scheme recently proposed by Herring and Herring ('74). Four points were defined on each cephalogram (fig. 2A): (1) the zygomaxillary root point (zrp), which is the most anterior origin of the superficial masseter muscle (Hylander, '75b; Carlson, '76); (2) the superior glenoid fossa point (sgf), defined as the intersect of the temporal surface of the glenoid fossa and a perpendicular to the maxillary occlusal plane through the mid-point of the mandibular condyle; (3) the mid-point of the mandibular condyle at centric occlusion (c); and (4) gonion (go). Two orientation planes were also defined: (1) the maxillary occlusal plane $(O P)$, a line tangential to the tip of the upper central incisor and the distobuccal cusp of the upper first molar; and (2) the maxillary occlusal plane perpendicular $(O P P)$, perpendicular to $O P$ through the superior glenoid fossa 
TABLE 1

Means and standard deviations for the functional angle $(\mathrm{F})$, lever arm length $(\mathrm{T})$, and length of the superficial masseter ( $\mathrm{L}$ ) during normal jaw rotation (Model $\mathrm{A}$ ) and with the condyle as a fixed axis of rotation (Model $\mathrm{B}$ )

\begin{tabular}{|c|c|c|c|c|c|c|}
\hline \multirow{2}{*}{$\begin{array}{l}\text { Rotation } \\
\text { model }\end{array}$} & \multicolumn{2}{|c|}{$\mathbf{F}_{1}$} & \multicolumn{2}{|c|}{$\mathrm{F}_{x t}$} & \multicolumn{2}{|c|}{$\mathrm{F}_{3}$} \\
\hline & $\bar{x}$ & s.d. & $\mathrm{x}$ & s.d. & $\mathrm{x}$ & s.d. \\
\hline A & 72.77 & 4.19 & 82.47 & 3.85 & 91.18 & 4.30 \\
\hline \multirow[t]{3}{*}{ B } & 72.77 & 4.19 & 91.40 & 4.36 & 103.77 & 5.70 \\
\hline & \multicolumn{2}{|c|}{$\mathrm{T}_{\mathrm{i}}$} & \multicolumn{2}{|c|}{$\mathbf{T}_{2}$} & \multicolumn{2}{|c|}{$\mathrm{T}_{3}$} \\
\hline & $\bar{x}$ & s.d. & $\bar{x}$ & s.d. & $\bar{x}$ & s.d. \\
\hline A & 3.19 & 0.25 & 2.94 & 0.28 & 2.60 & 0.31 \\
\hline \multirow[t]{3}{*}{ B } & 3.19 & 0.25 & 2.72 & 0.26 & 2.38 & 0.27 \\
\hline & \multicolumn{2}{|c|}{$\mathrm{L}_{\iota_{1}}$} & \multicolumn{2}{|c|}{$\mathrm{L}_{2}$} & \multicolumn{2}{|c|}{ L } \\
\hline & $\bar{x}$ & s.d. & $\bar{x}$ & s.d. & $\bar{x}$ & s.d. \\
\hline A & 4.83 & 0.49 & 5.30 & 0.49 & 5.53 & 0.47 \\
\hline B & 4.83 & 0.49 & 5.77 & 0.50 & 6.37 & 0.49 \\
\hline
\end{tabular}

Subscripts indicate the mandibular position at which the data were recorded: 1 , occlusal/rest position; $2.3 \mathrm{~cm}$ incisal opening; 3 , maximum gape

TABLE 2

Percent change in functional angle $(\mathrm{F})$, lever arm length $(\mathrm{T})$, and superficial masseter length (L) between the occlusal-rest position (Position 1), $3 \mathrm{em}$ incisal open bite (Position 2), and maximum gape (Position 3) for Model A (normal jaw rotation) and Model $B$ (rotation about a fixed axis at the condyles)

\begin{tabular}{crrr}
\hline $\begin{array}{c}\text { Rotation } \\
\text { model }\end{array}$ & $\mathrm{F}_{2}-\mathrm{F}_{3}$ & $\mathrm{~F}_{1}-\mathrm{F}_{3}$ & $\mathrm{~F}_{2}-\mathrm{F}_{3}$ \\
\hline $\mathrm{A}$ & 13.3 & 25.3 & 12.0 \\
$\mathrm{~B}$ & 25.6 & 42.6 & 17.0 \\
& $\mathrm{~T}_{1}-\mathrm{T}_{2}$ & $\mathrm{~T}_{2}-\mathrm{T}_{3}$ & $\mathrm{~T}_{2}-\mathrm{T}_{3}$ \\
\hline $\mathrm{A}$ & -7.8 & -18.5 & -10.6 \\
$\mathrm{~B}$ & -14.7 & -24.4 & -9.7 \\
& $\mathrm{~L}_{1}-\mathrm{L}_{2}$ & $\mathrm{~L}_{1}-\mathrm{K}_{3}$ & $\mathrm{~L}_{2}-\mathrm{L}_{3}$ \\
\hline $\mathrm{A}$ & 9.7 & 14.5 & 4.8 \\
$\mathrm{~B}$ & 19.5 & 31.9 & 12.4 \\
\hline
\end{tabular}

point (sgf). Three variables were constructed from these cephalometric points (fig. 2B):

(1) Functional angle $(F)$ : the angle formed at the superior glenoid fossa point (sgf) by arms extending to the zygomaxillary root point (zrp) and gonion (go).

(2) Lever arm length ( $T$ ). the perpendicular distance to the superior glenoid fossa point (sgf) from a line connecting the zygomaxillary root point erpj and gonion (go). Torque, or the magnitude of twist about a center of rotation, is a function of lever arm length and the magnitude of the force exerted by the muscle such that Torque $=$ force $\times$ lever arm length

(3) Superficial masseter length ( $L)$ : the distance from the \%ygomaxillary root point ( $\mathrm{rrp}$ ) to gonion (go).

Rotation model A (fig. 3A)

Cephalograms taken with the mandible in centric occlusion were traced onto acetate film, and the positions of the bone markers were noted. Tracings of the remaining two positions were made on the same film following superimpositioning of the cranial outline and implants. Thus, the mandible was the only element which varied spatially over the three views.

\section{Rotation model $B$ (fig. 3B)}

A second series of tracings was made for each animal. In this case, however, the registration was not made on the cranial outline and markers, but the maxillary occlusal plane $(O P)$ and the maxillary occlusal plane perpendicular $(O P P)$. The net effect of this registration procedure was to maintain the condyle artificially in its centric occlusal position within the glenoid fossa at all three mandibular positions, thus giving the impression that the mandible was functioning as a simple hinge joint about a fixed axis.

\section{RESULTS}

During normal jaw opening, where the mandibular condyles rotate and are translated anteriorly-inferiorly (Model A), three distinct results obtain (tables 1, 2). At Position 2 ( $3 \mathrm{~cm}$ incisal opening) the mean increase in the functional angle is $13.3^{\circ}$ from the rest-occlusal position (Position 1). This increase in functional angle reaches approximately $20^{\circ}(25.3 \%)$ by Position 3 (maximum gape). 
1

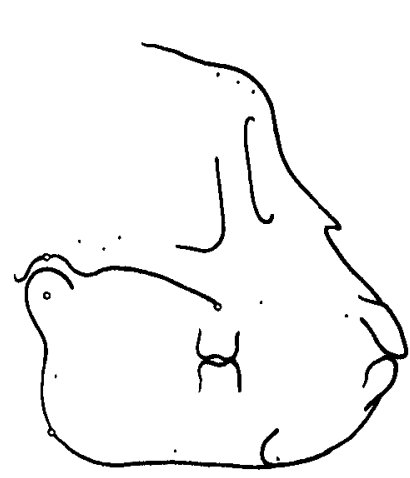

2

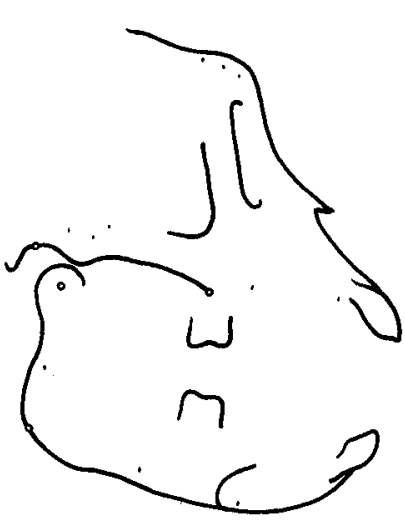

MODE L A

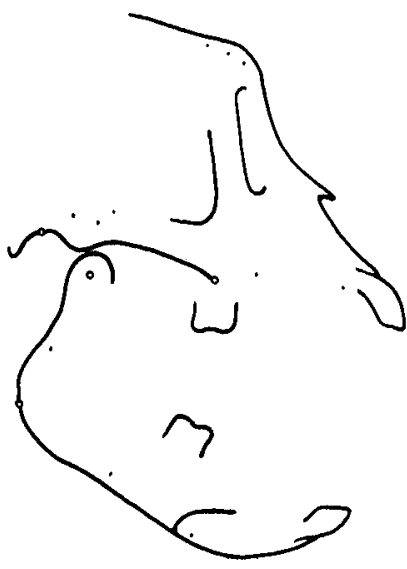

1

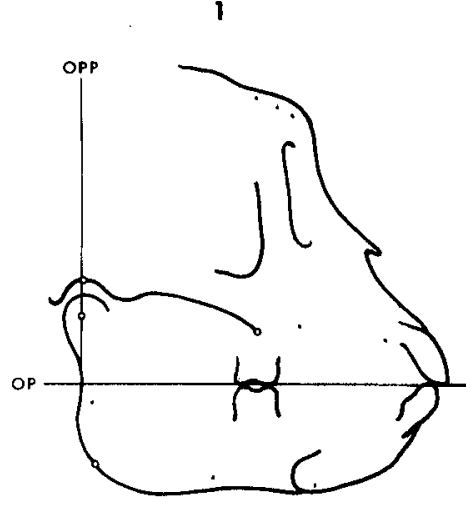

2

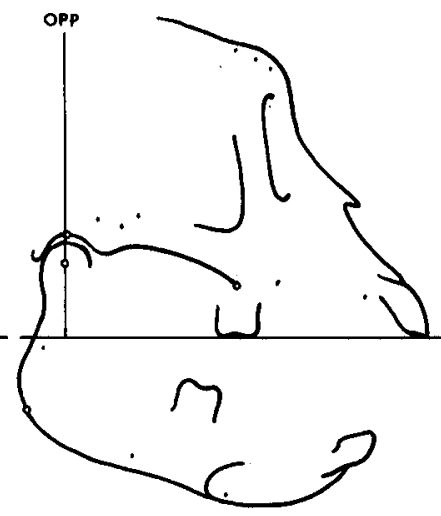

3

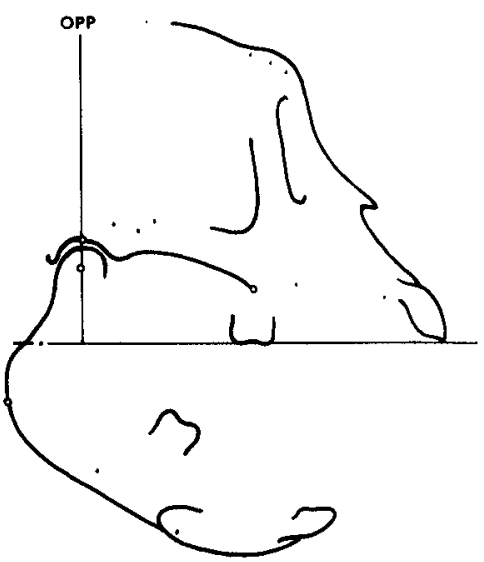

MODEL B

Fig. 3 Tracings of lateral cephalograms of three static mandibular positions during symmetrical rotation: 1, occlusal-rest position; $2,3 \mathrm{~cm}$ open bite; 3 , maximum gape. Model A: normal jaw rotation. Cephalograms were superimposed using cranial outlines and implants, and the mandible was traced as it rotated from the first through the third positions. Model B: jaw rotation about condyles artificially fixed within the glenoid fossae. Cephalograms were superimposed initially using cranial outlines and implants as above. However, tracings of mandibular rotation through Positions 2 and 3 were obtained by reorienting along the maxillary occlusal plane (OP), such that the maxillary occlusal plane perpendicular (OPP) passed through the condyle (c) and the superior glenoid fossa point (sgf). This procedure artificially maintained the condyles within their normal occlusal-rest position and did not allow them to be translated during jaw rotation.

The decrease in lever arm length is almost linear with jaw opening for the positions studied. There is a $7.8 \%$ decrease in the length of the lever arm for the superficial masseter muscle at Position 2 relative to the occlusal- rest position, and a decrease of $18.5 \%$ at maximum gape. Superficial masseter length follows a similar course, stretching by $9.7 \%$ $\left(\mathrm{L}_{1.10}\right)$ at Position 2 a d $14.5 \%\left(\mathrm{~L}_{1.14}\right)$ at Position 3. 


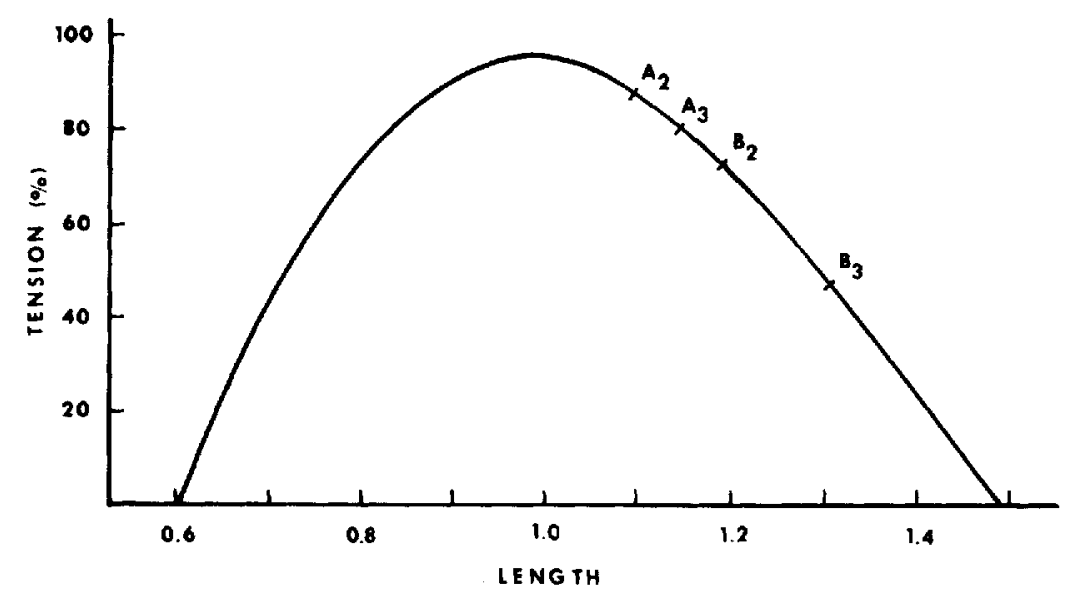

\begin{tabular}{|c|c|c|c|}
\cline { 2 - 4 } \multicolumn{1}{c|}{} & \multicolumn{3}{c|}{ MAXIMUM } \\
\cline { 2 - 4 } & \multicolumn{3}{c|}{ PRODUCIBLE TENSION $(\%)$} \\
\hline A & 100 & 2 & 3 \\
\hline B & 100 & 70 & 45 \\
\hline
\end{tabular}

Fig. 4 Length-tension diagram for a whole muscle demonstrating the effect of stretching the superficial masseter muscle during jaw rotation for Models $A$ and $B$. Note that at $3 \mathrm{~cm}$ incisal open bite during normal symmetrical jaw rotation $\left(A_{2}\right)$ reduction in maximum producible tension is only about $12 \%$, and that at maximum gape $\left(A_{3}\right)$ there is approximately a $20 \%$ decrease. In the absence of condylar translation during jaw rotation reduction in producible tension at Position $2\left(B_{2}\right)$ is $30 \%$ and a substantial $55 \%$ decrease at maximum gape $\left(B_{3}\right)$.

The same general relationships naturally hold for Model B, where the condyles are assumed to be fixed within the glenoid fossae during all phases of mandibular rotation. In this case, however, the effects of the same amounts of jaw opening as in Model A are substantially more dramatic. For example, the functional angle increases by $25.6 \%$ at $\mathrm{Po}$ sition 2 , and by $42.6 \%$ by Position 3 during jaw rotation with the condyles as fixed axes. This represents a $12.3 \%$ and a $17.3 \%$ greater increase in $F$ than in Positions 2 and 3, respectively, during normal rotation.

The effects of this difference in functional angle in Model B are manifold with respect to lever arm length and muscle excursion. Lever arm length decreases by $14.7 \%$ at Position 2 and $24.4 \%$ at Position 3 from the occlusal-rest position, decreases, respectively, of $6.9 \%$ and $5.9 \%$ greater than during normal rotation. The relative increase in the length of the superficial masseter muscle is even more pronounced. The muscle is stretched $19.5 \%$ beyond its resting length $\left(\mathrm{L}_{1.19}\right)$ at Position 2 , and $31.9 \%\left(\mathrm{~L}_{1.32}\right)$ at maximum gape. This represents a $9.8 \%$ and $17.4 \%$ greater superficial masseter excursion in Model B relative to the normal jaw rotation in Model A for Positions 2 and 3 , respectively.

\section{DISCUSSION}

The three variables examined in this study are necessarily closely interrelated. Normal mandibular rotation during the opening phase causes a posterior translation of the mandible, thereby increasing the functional angle. This, in turn, causes an increase in the length of superficial masseter from its resting length $\left(L_{1.0}\right)$, and a decrease in the relative torque of superficial masseter. The significance of this action for jaw function from a mouth-open position, however, depends in large part on the presence of compensatory factors which might decrease the adverse effects of jaw opening on relative excursion and torque. 
Change in the functional angle during jaw opening is clearly dependent on the degree of opening of the mouth and the location of the axis of mandibular rotation. During normal jaw opening in most primates (Model A) the anterior translation of the condyles permits the mouth to be opened without radically increasing the functional angle, as would occur if the jaw functioned as a simple hinge about fixed condyles (Model B). In the latter case the mandibular corpus is translated posteriorly during jaw opening to a much greater extent than during normal rotation.

The effect of minimizing posterior translation of the mandible during opening is apparent in the consideration of relative torque. It has been demonstrated that the decrease in lever arm length for superficial masseter is minimized by anterior translation of the condyles during rotation to an open position. The magnitude of the differences in lever arm length between normal rotation and rotation about fixed condyles suggests that this is of major significance in the consideration of jaw function and relative torque.

Consideration of the excursion of superficial masseter during Model A and Model B types of rotation demonstrates that this factor is probably most significant for understanding primate jaw mechanics. There is approximately a $10 \%$ difference in the stretch of superficial masseter between normal rotation and rotation about a fixed axis at $3 \mathrm{~cm}$ incisal opening and a 17\% difference at maximum gape. These differences for superficial masseter function becomes most apparent when excursion length in each case is considered in terms of the length-tension relationship (fig. 4).

During normal jaw function the $9.7 \%$ increase in superficial masseter leng th from the rest position to Position 2 results in a decrease in maximum producible tension of approximately $12 \%$, while at maximum gape it is reduced by $20 \%$. In other words, complete tetanic stimulation of the superficial masseter muscle with a $3 \mathrm{~cm}$ incisal open bite will result in a maximum producible tension $88 \%$ of that producible at resting length. Stimulation at maximum gape would result in a maximum of $80 \%$ producible tension.

During jaw function about fixed condyles, on the other hand, the resultant maximum producible tension is dramatically decreased because of the increased excursion of the superficial masseter muscle. Maximum produci- ble tension upon complete tetanic stimulation is reduced by approximately $30 \%$ at Position 2 , and by approximately $55 \%$ at Position 3 . Thus, maximum producible tetanic tension with the mouth open $3 \mathrm{~cm}$ incisally is only $70 \%$ of that producible at resting length, while at maximum gape it is only $45 \%$. Furthermore, according to Gordon et al. ('66), 30\% excursion for most skeletal muscles may result in some degree of detachment of the muscle from its skeletal origin and insertion. The magnitude of the differences between normal mandibular rotation and rotation about fixed condyles thus indicates that minimizing the excursion of superficial masseter during opening may be a major function of the mechanics of the TMJ.

\section{Implications}

It is well-established that TMJ morphology is closely related to jaw function (Moffett, '66; Turnbull, '70; McNamara, '72; Noble, '73). However, investigations of this relationship most often consider only relative muscle torque, usually using the dry skull or perfused specimens in which normal mandibular positions are difficult or impossible to ascertain. As a result, analysis of other parameters of muscle function in the masticatory complex, such as the mechanics of muscle contraction, has received insufficient attention. Even those analyses which attempt to consider factors other than muscle torque have not properly considered the effect of TMJ mechanics on muscle function.

In a theoretical-comparative analysis of superficial masseter and gape in mammals, for example, Herring and Herring ('74) consider excursion to be a potential factor influencing mandibular morphology. Their analysis is limited to a geometric consideration of jaw mechanics using dry skulls of several mammalian forms, however, and they do not take TMJ mechanics into account. All forms are analyzed with the mandibular condyles held in a fixed position within the glenoid fossae. Thus, while the results presented here support Herring and Herring's conclusion that jaw morphology in many mammalian forms may be the result of adaptation for gape, their analysis must be considered incomplete, particularly with respect to the primates.

The view that the variable axis of mandibular rotation in primates, including man, is inconsequential to the analysis of jaw function (Hylander, '75a) is valid only if muscle torque alone is considered. Detailed understanding of 
TMJ mechanics, including consideration of condylar translation during opening and closure phases, is necessary if other parameters of jaw function are to be considered.

The present study suggests that morphological-biomechanical adaptations to reduce muscle excursion are of critical importance in efficient jaw function. More specifically, the anterior-inferior translation of the mandibu. lar condyles along the posterior surface of the articular eminence during jaw opening functions to minimize both the reduction in relative torque and the increase in excursion of the superficial masseter muscle, thereby maintaining optimal potential for exerting maximum tension during jaw closure on either side of the jaw as well as during incisal biting. Viewed in terms of the length-tension relationship for muscle contraction, therefore, this latter parameter may be critical to the efficient function of the jaws in primates and thus of major adaptive significance in the evolution of the primate masticatory complex. Further evaluation of this hypothesis should be undertaken (1) by similar analysis of each of the primary elevators of the mandible in macaques and other primates during both symmetrical jaw rotation and mastication associated with lateral jaw movement, and (2) by detailed analysis of fiber geometry and fiber type for each of the muscles of mastication in primates.

\section{ACKNOWLEDGMENTS}

Appreciation is extended to Doctors J. A. McNamara, L. W. Graber, R. G. Behrents, L. Maxwell, W. Jungers, T. Calhoun and E. Johnson for their comments and suggestions during the course of the research. Sincere thanks also to Ms. Jody Ungerleider for her help with radiographic techniques and to Ms. Donna Monroe for her editorial assistance and typing of the final draft.

\section{LITERATURE CITED}

Barbenel, J. C. 1972 The biomechanics of the temporomandibular joint: A theoretical study. J. of Biomech., 5 : 251-256.

Brill, N., and G. Tryde 1974 Physiology of mandibular posi. tions. In: Frontiers of Oral Physiology. Vol. I Physiology of Mastication. Y. Kawamura, ed. Karger, Basel, pp. 200 . 237.

Carlson, D. S. 1976 Tempora] variation in prehistoric Nubian crania. Am. J. Phys. Anthrop. 45: 467-484.

Carlson, F. D., and D. R. Wilkie 1974 Muscle Physiology. Prentice-Hall, Englewood Cliffs.

Crompton, A. W. 1963 The evolution of the mammalian jaw. Evolution 17: 431-439.
Davis, D. D. 1955 Masticatory apparatus in the spectacled bear Tremarctos ornatus. Fieldiana Zoology, 37: 25-46.

Du Brul, E. L. 1974 Origin and evolution of the oral apparatus. In: Frontiers of Oral Physiology. Vol. I, Physiology of Mastication. Y. Kawamura, ed. Karger, Basel, pp. 1.30.

Gans, C., and W. J. Bock 1965 IV. The functional significance of muscle architecture -a theoretical analysis. Rev. Anat. Embryol. Cell Biol., 38: 115-142.

Garnick, J. J., and S. P. Ramfjord 1962 Rest position. J. Prosth. Dent., 12; 895-911.

Gibbs, C. H., T. Messerman, J. B. Reswick and H. J. Derda 1971 Functional movements of the mandible. J. of Prosth. Dent., 26: 604-620.

Gilbe, G. V. 1973 A comparison of the disc in the craniomandibular joint of three mammals. Acta Anat., 86: 384409.

Gingerich, P. D. 1971 Functional significance of mandibular translation in vertebrate jaw mechanics. Postilla, I52: 3-10.

Gordon, A. M., A. F. Huckley and F. J. Julian 1966 The variation in isometric tension with sarcomere length in vertebrate muscle fibers. J. Physiol. (Iondon), 184: 170192.

Graber, T. M. 1972 Orthodontics: Principles and Practice. Third ed. W. B. Saunders, Philadelphia.

Graf, H. 1975 Occlusal forces during function. In: Occlusion: Research in Form and Function. N. Rowe, ed. Univ. of Michigan School of Dentistry, Ann Arbor, pp. 112-119.

Grant, P. D. 1973 Biomechanical significance of the instantaneous center of rotation: The human temporomandibular joint. J. Biomech., 6: 109.113.

Griffin, C. J., and R. Malor 1974 An analysis of mandibular movement. In: Frontiers of Oral Physiology. Vol. I, Phys iology of Mastication. Y. Kawamura, ed. Karger, Basel, pp. 160-199.

Herring, S. W., and S. E. Herring 1974 The superficial masseter and gape in mammals. Am. Natural., 108: 561 . 576 .

Hiiemae, K. M. 1971 The structure and function of jaw muscles in the rat (Rattus norvegicus $L$.). III. The mechanics of the muscles. Zool. J. Linn. Soc., 50: 111-132.

Hiiemae, K. M. and R. F. Kay 1973 Evolutionary trends in the dynamics of primate mastication. In: Craniofacial Biology of Primates. Vol. 3. Symp. IVth Int. Cong. Primat. M. R. Zingeser, ed. Karger, Basel, pp. 28-64.

Hill, A. V. 1953 The mechanics of active muscle. Proc. Roy. Soc., $B, 141$ : 104-117.

Hjortsjö, C. H. 1955 Studies on the mechanics of the temporomandibular joint. Lund 1951-1954. Lund Universitets Arsskrift. N.F. Avd. 2. Bd. 51, Nr. 2.

Hoshi, H. 1971 Comparative morphology of the mammalian mandible in relation to food habit. Okajimas Fol Anat. Jap. 48: 333-345.

Hylander, W. L. 1975a The human mandible: Lever or link? Am. J. Phys. Anthrop., 43: 227-242.

$1975 \mathrm{~b}$ The adaptive significance of Eskimo cra niofacial morphology. In: Oro-Facial Growth and Development. A. A. Dahlberg and T. M. Graber, eds. Mouton, pp. 129-169.

Isaacson, R. J., R. L. Christiansen, C. A. Evans and R. A. Riedel 1975 Research on variation in dental occlusion. Am. J. Orthod., 68: 241-255.

Kay, R. F., and K. M. Hiiemae 1974 Jaw movement and tooth use in recent and fossil primates. Am. J. Phys. Anthrop., 40: 227-256.

Latif, A. 1957 An electromyographic study of the temporal muscle in normal persons during selected positions 
and movements of the mandible. Amer. J. Orthodont., 43 $577-591$.

McNamara, J. A., Jr. 1972 Neuromuscular and Skeletal Adaptations to Altered Orofacial Function. Center for Human Growth and Development, Ann Arbor, Craniofacial Growth Series Monograph No. 1.

1974 The role of muscle and bone interaction during craniofacial growth. In: Control Mechanisms in Craniofacial Growth. J. A. McNamara, ed. Center for Human Growth and Development, Ann Arbor, Craniofacial Growth Series Monograph No. 3, pp. 51-74.

Moffett, B. 1966 The morphogenesis of the temporomandibular joint. Am. J. of Orthodont., 52: 401-415.

Møller, E. 1966 The chewing apparatus: An electromyographic study of the action of the muscles of mastication and its correlation to facial morphology. Acta. Physio. Scand., (Suppl.) 69: 280.

1974 Action of the muscles of mastication. In: Frontiers of Oral Biology. Vol. I, Physiology of Mastication. Y. Kawamura, ed. Karger, Basel, pp. 121-159.

Moss, M. L. 1959 Functional anatomy of the temporomandibular joint. In: Disorders of the Temporomandibular Joint. L. Schwartz, ed. W. B. Saunders, Philadelphia, pp. $73-88$.

Noble, H. W. 1973 Comparative functional anatomy of temporomandibular joint. Oral Sci. Rev., 2: 3-28.

Ramfjord, S. P., and M. M. Ash, Jr. 1971 Occlusion. W. B. Saunders, Philadelphia.

Ramsey, R. W. 1955 Analysis of contraction of skeletal muscle. Am. J. Physiol., 181: 688-702.

1960 Some aspects of the biophysics of muscle. In: Structure and Function of Muscle. Vol. II, Biochemis- try and Physiology. G. Bourne, ed. Academic Press, N.Y., pp. 303-358.

Ramsey, R. W., and S. F. Street 1940 The isometric length tension diagram of isolated muscle fibers of the frog. J. Cell. Comp. Physiol., 15: 11-34.

Rayne, J., and G. N. C. Crawford 1972 The relationship between fibre length, muscle excursion and jaw movements in the rat. Archs. Oral Biology, 17: 859-872.

Ricketts, R. M. 1975 Mechanisms of mandibular growth: A series of inquiries on the growth of the mandible. In: Determinants of Mandibular Form and Growth. J. A. McNamara, Jr., ed. Center for Human Growth and Development, Ann Arbor, Craniofacial Growth Series Monograph No. 4, pp. 77-100.

Scapino, R. P. 1972 Adaptive radiation of mammalian jaws. In: Morphology of the Maxillo-Mandibular Apparatus. G. H. Schumacher, ed. Thieme, Leipzig, pp. 33 . 39.

Smith, J. M., and R. J. G. Savage 1959 The mechanics of mammalian jaws. School Sci. Rev., 141: 289-301.

Solow, B. 1966 The Patterns of Craniofacial Associations. Acta. Odont. Scand., 24: Suppl. 46.

Stern, J. T. 1974 Biomechanical significance of the instantaneous center of rotation: the human temporomandibular joint. J. Biomech., 7: 109.

Turnbull, W. D. 1970 Mammalian Masticatory Apparatus. Fieldiana: Geology, 18.

Wolpoff, M. H. 1975 Some aspects of human mandibular evolution. In: Determinants of Mandibular Form and Growth. J. A. McNamara, ed. Center for Human Growth and Development, Ann Arbor, Craniofacial Growth Series Monograph No. 4, pp. 1-64. 